\title{
Social Inequities Between Prenatal Patients in Family Medicine and Obstetrics and Gynecology with Similar Outcomes
}

\author{
Michael Partin, MD, Ana Sanchez, MD, Jessica Poulson, BS, Arthur Berg, PhD, \\ Jessica Parascando, MPH, and Sarah Ines Ramirez, MD
}

Introduction: Family Medicine (FM) physicians play a vital role in caring for vulnerable populations across diverse practice settings. The significant decline in FM physicians performing deliveries compounds the estimated shortage of 9000 prenatal care providers expected by 2030.

This study investigated the social risk profile, as characterized by social determinants of health, of patients receiving prenatal care from FM versus Obstetrics and Gynecology (OB/Gyn) providers.

Methods: Retrospective chart review of patients receiving prenatal care between 2015 to 2018 at Penn State Health Hershey Medical Center comparing social determinants of health between FM and OB/Gyn.

Results: A total of 487 patient charts were reviewed with final analysis completed on 215 charts from each cohort. When compared with $\mathrm{OB} / \mathrm{Gyn}$, prenatal patients cared for by $\mathrm{FM}$ were more likely to be younger (27 vs 29 years old; $P<.0001)$, African American $(28 \%$ vs $8 \% ; P<.0001)$, single $(52 \%$ vs $37 \% ; P<.01)$, have high school or less education $(67 \%$ vs $49 \% ; P<.01)$, use Medicaid $(46 \%$ vs $23 \%$; $P<.0001)$, and use tobacco during pregnancy $(17 \%$ vs $8 \% ; P<.01)$. In addition, FM patients had a lower rate of total Cesarean-sections (C-section), including primary and repeat, when compared with OB/Gyn (23\% vs 32\%; $P=.04)$.

Conclusions: Our work demonstrates that when compared with $0 B / G y n$ at our institution, FM physicians provide care to a cohort of patients with an increased burden of social risk without compromise to care as evidenced by a lower $\mathrm{C}$-section rate and similar gestational age at delivery. ( $\mathrm{J}$ Am Board Fam Med 2021;34:181-188.)

Keywords: Cesarean Section, Cohort Studies, Family Medicine, Gynecology, Internship and Residency, Obstetrics, Pregnancy, Prenatal Care, Retrospective Studies, Social Determinants of Health

\section{Introduction}

Family Medicine (FM) physicians play a vital role in caring for vulnerable patient populations with opportunities to provide holistic and cost-effective care for entire family units across diverse practice

This article was externally peer reviewed.

Submitted 8 June 2020; revised 28 September 2020; accepted 29 September 2020.

From the Department of Family and Community Medicine, College of Medicine, Penn State Hershey, PA (MP, JP, SIR); Department of Family Medicine, Penn Medicine, Lancaster General Health, Lancaster, PA (AS); College of Medicine, Penn State, Hershey, PA (JP); Department of Public Health Sciences, College of Medicine, Penn State, Hershey, PA (AB).

\section{Funding: None.}

Conflict of interest: None.

Corresponding author: Sarah Ines Ramirez, MD, Department of Family and Community Medicine, Penn State Health Hershey Medical Center, 500 University Drive; HP 11, Hershey, PA 17033 (E-mail: sramirez2@pennstatehealth.psu. edu). settings, including obstetrics. ${ }^{1,2}$ With an estimated shortage of 9000 prenatal care (PNC) providers by 2030, the need for an increase in the number of FM physicians who provide PNC is glaring. ${ }^{3,4}$ Longitudinal survey data have noted a significant decline in $\mathrm{FM}$ physicians performing deliveries from $44 \%$ in $1982 \%$ to $18 \%$ in $2018 .{ }^{5}$ In addition, analysis of proportion of PNC visits performed by FM physicians between 1995 and 2004 showed a decrease from $11.6 \%$ to $6.1 \% .{ }^{6}$ Compounding this shortage is the fact that Obstetrics and Gynecology (OB/ Gyn) residency positions continue to lag behind the increase in the United States adult female population as well as a noted increase in OB/Gyn residency graduates pursuing fellowships that do not include routine PNC. ${ }^{7,8}$ The resulting landscape affords opportunities for FM physicians to provide such care, especially to patient populations with 
increased social complexity, which is often defined through the lens of social determinants of health $(\mathrm{SDoH}){ }^{9,10}$

$\mathrm{SDoH}$ are widely acknowledged as powerful social and environmental forces that affect health outcomes and act as indicators of health risk, including economic stability, neighborhood environment, health and health care, education, and community context. ${ }^{9,11,12} \mathrm{SDoH}$ are particularly relevant to PNC as they affect the health outcomes of mothers and infants. For instance, the rate of maternal morbidity and mortality is higher for women in the United States than in other developed countries, with African-American women at the highest risk of these poor outcomes. ${ }^{13,14}$ Furthermore, attending fewer than 10 prenatal visits and having less than a high school education is associated with increased maternal mortality among women of childbearing age. ${ }^{15}$ As mothers and infants share a background of socioeconomic status, ethnicity, and living area, infants have similar health disparity outcomes reflected in low birth weight and preterm delivery for disadvantaged groups. ${ }^{16-18}$

Despite the known association between maternal and infant morbidity and mortality with $\mathrm{SDoH}$, there is a paucity of research looking at the $\mathrm{SDoH}$ for prenatal patients cared for by FM providers as compared with $\mathrm{OB} / \mathrm{Gyn}$ providers. FM physicians receive intentional training aimed at providing comprehensive care in a continuity model that not only identifies and treats isolated disease, but ultimately focuses on the individual patient and the numerous ways in which SDoH impact families. ${ }^{19,20}$ Therefore, FM physicians are poised to care for diverse, vulnerable patient populations, especially in the setting of PNC. This study aimed to evaluate the social complexity, as defined by SDoH, of prenatal patients cared for by FM as compared with $\mathrm{OB} / \mathrm{Gyn}$ at our institution. SDoH elements studied were broadly classified into demographics, social history, routine PNC received, and pregnancy outcomes.

\section{Methods}

This retrospective chart review was approved by the Penn State College of Medicine Institutional Review Board through expedited review. We identified patients receiving PNC between January 1, 2015 and December 31, 2018 at Penn State Health FM and $\mathrm{OB} / \mathrm{Gyn}$ clinics. This pool of eligible patients was identified using pregnancy-related International
Classification of Disease-10 (ICD-10) codes. Patients were included in the study if they were female, between the ages of 12 and 51 years, and if they received their PNC by FM or OB/Gyn at our institution. Patients were excluded if they transferred care between FM and $\mathrm{OB} / \mathrm{Gyn}$ or transferred to the Maternal Fetal Medicine service. At our institution, FM provides $\mathrm{PNC}$ at 8 practice sites and OB/Gyn at 3 practice sites with care being provided by faculty and residents. In addition, $\mathrm{OB} / \mathrm{Gyn}$ practice sites have Nurse Practitioners and Nurse Midwives providing PNC as well. Access to nutritionists is available on site to OB/Gyn pa-tients, and FM patients can be referred in to receive these services as well. Social work services are available to both departments. The FM practice model included 1 provider seeing the patient for the entirety of PNC with the exception of those assigned to FM residents as continuity patients. OB/Gyn rotated providers based on availability. Neither cohort utilized group visits or centering care models.

Chart review elements included employment and health insurance status, highest education level obtained, residential ZIP code, race, marital status, primary language spoken, gestational age at initial prenatal visit, past medical history, tobacco/alcohol/recreational drug use before and during pregnancy, interconception period, use of birth control at conception, rates of intimate partner violence, number of prior pregnancies (gravidity), prevalence of sexually transmitted infections (sexually transmitted infection), adherence to immunization schedule, and adherence to prenatal visit schedule as defined by the American Congress of Obstetrics and Gynecology (ACOG) prenatal visit schedule. ${ }^{21}$

The interconception period was defined as the number of months between the date of delivery for the previous pregnancy and the date of last menstrual period corresponding to the subsequent pregnancy. ${ }^{22}$ Adherence to immunization schedule was defined as whether both influenza and Tdap vaccines were received during pregnancy. Pregnancy outcome data included birth weight of infants, mode of delivery, and gestational age at delivery. The majority of the data were obtained through chart review of standardized ACOG prenatal record forms used for all patients at our institution (FM and $\mathrm{OB} / \mathrm{Gyn}$ ) with additional information supplemented from office visit documentation and delivery operative reports.

A pilot chart review of $47 \mathrm{FM}$ and $46 \mathrm{OB} / \mathrm{Gyn}$ patients was conducted to identify an adequate 
Figure 1. Study population flow diagram. Abbreviations: FM, Family Medicine; OB/Gyn, Obstetrics and Gynecology; ICD, International Classification of Diseases.

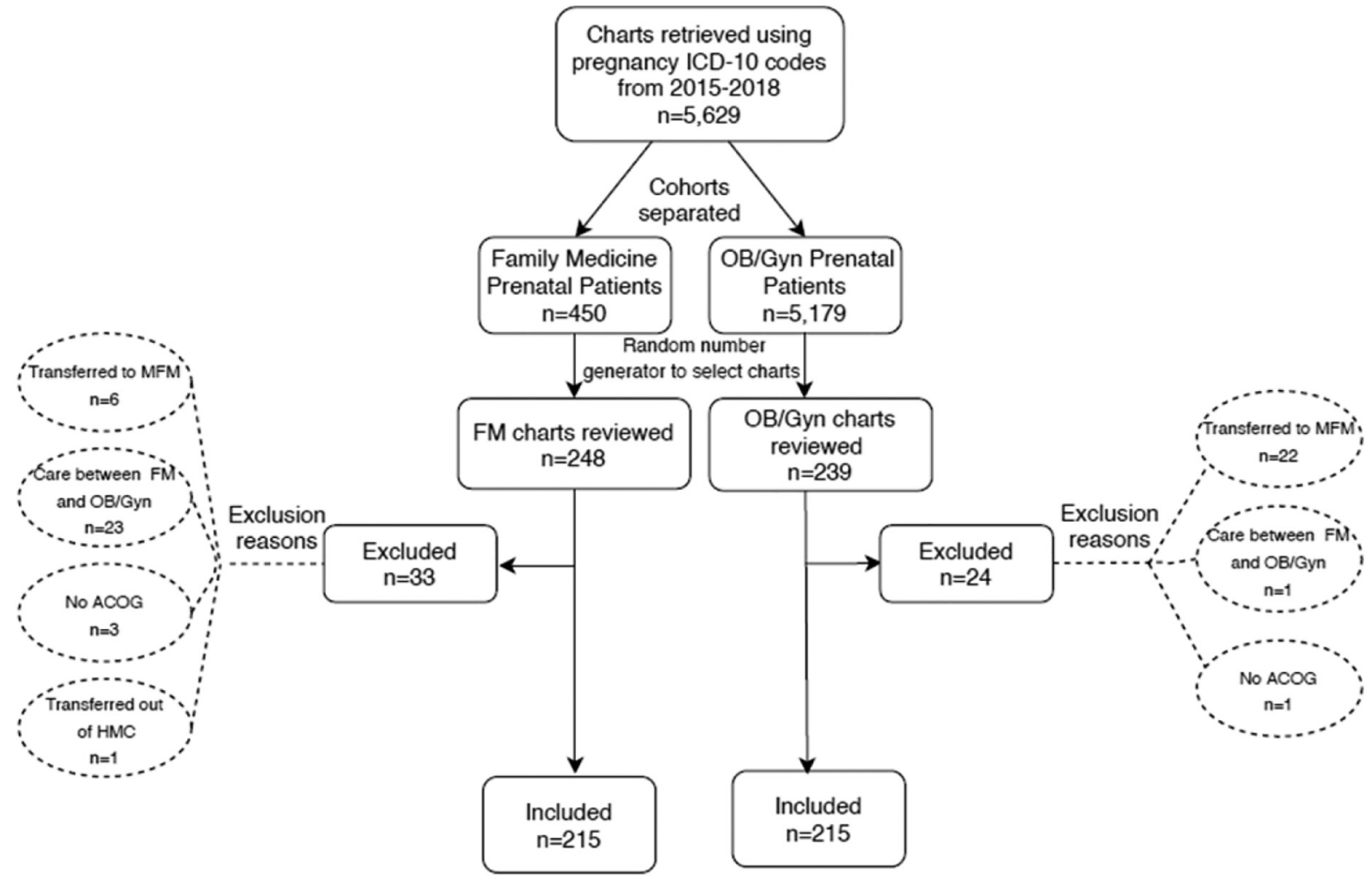

sample size for a larger study. Focusing on the primary variables of Body Mass Index, age, race, smoking status, and Medicaid use, and basing the anticipated effect sizes on the pilot data, a sample size for each cohort was determined to be 212 patients to achieve adequate power with an $\alpha$ level of 0.01 . Charts were selected for review using a random number generator with minimum set to 1 and maximum set to total number of charts for each cohort. Various software programs were used in the statistical analysis including R (R Foundation for Statistical Computing, Vienna, Austria), SPSS (SPSS, Inc., Chicago, IL), and Microsoft Excel (Microsoft, Redmond, WA). The $\mathrm{c}^{2}$ test was used to analyze associations with categorical variables. The nonparametric Mann-Whitney $U$-test was used to analyze associations with continuously distributed data.

\section{Results}

\section{Study Population}

An initial 5629 patients were identified from both the FM and OB/Gyn cohorts between January 1.
2015 and December 31, 2018. A total of 487 patient charts were ultimately reviewed with 248 charts from the FM cohort and 239 from OB/Gyn. Of these, 33 were excluded from the FM cohort and 24 from the $\mathrm{OB} / \mathrm{Gyn}$ cohort. Exclusion reasons included transferring care between FM and OB/ Gyn $(n=24)$, transfer to Maternal Fetal Medicine $(\mathrm{n}=28)$, transfer to outside institution $(\mathrm{n}=1)$, and no ACOG documentation available $(n=4)$. Final analysis was completed on 215 patient records per cohort (Figure 1).

\section{Demographics}

The overall characteristics of the study population are summarized in Table 1. FM provided care for more African American patients (28\% vs $8 \%$; $P<.00001)$ and fewer white patients when compared with the OB/Gyn cohort (51\% vs $72 \%$; $P<.00001)$. Patients from the FM cohort had a higher proportion of less than or the equivalent of high school education ( $67 \%$ vs $49 \% ; P<.01)$. FM patients were more likely to be single (52\% vs $37 \%$; $P<.01)$ when compared with OB/Gyn. In addition, the percentage of employed patients within the FM 
Table 1. Characteristics of Prenatal Patients Cared for by FM or OB/Gyn between January 2015 and December 2018

\begin{tabular}{|c|c|c|c|c|}
\hline & Overall & Family Medicine & Obstetrics and Gynecology & $P$ Value \\
\hline Overall, No. (\%) & $430(100.0)$ & $215(50.0)$ & $215(50.0)$ & - \\
\hline Age, year, mean (SD) & $27.7(5.68)$ & $26.6(5.7)$ & $28.6(5.6)$ & $<.01$ \\
\hline Race, No. (\%) & & $215(50.0)$ & $215(50.0)$ & \\
\hline Asian & $8(1.9)$ & $3(1.4)$ & $5(2.3)$ & .48 \\
\hline African American & $79(18.4)$ & $61(28.4)$ & $18(8.4)$ & $<.01$ \\
\hline White & $265(61.6)$ & $110(51.2)$ & $155(72.1)$ & $<.01$ \\
\hline Other & $66(15.3)$ & $32(14.9)$ & $34(15.8)$ & .83 \\
\hline Education, No. (\%) & & $215(50.0)$ & $215(50.0)$ & \\
\hline Grade school & $20(4.7)$ & $5(2.3)$ & $15(7.0)$ & $<.05$ \\
\hline High school & $139(32.3)$ & $74(34.4)$ & $65(30.2)$ & .35 \\
\hline GED & $6(1.4)$ & $2(0.9)$ & $4(1.9)$ & 1.00 \\
\hline Trade school & $14(3.3)$ & $5(2.3)$ & $9(4.2)$ & .26 \\
\hline College & $78(18.1)$ & $28(13.0)$ & $50(23.3)$ & $<.01$ \\
\hline Postgraduate & $34(7.9)$ & $7(3.3)$ & $27(12.6)$ & $<.01$ \\
\hline Unknown & $141(32.8)$ & $94(43.7)$ & $47(21.9)$ & $<.01$ \\
\hline Employment, No. (\%) & & $215(50.0)$ & $215(50.0)$ & \\
\hline Employed & $283(65.8)$ & $131(61.0)$ & $152(70.7)$ & $<.05$ \\
\hline Unemployed & $89(20.7)$ & $39(18.1)$ & $50(23.3)$ & .19 \\
\hline Unknown & $58(13.5)$ & $45(20.9)$ & $13(6.0)$ & $<.01$ \\
\hline Unemployed stratified, No. (\%) & $88(100.0)$ & $38(43.2)$ & $50(56.8)$ & \\
\hline Homemaker & $38(43.2)$ & $18(47.3)$ & $20(40.0)$ & .49 \\
\hline Student & $22(0.3)$ & $13(34.2)$ & $9(18.0)$ & .08 \\
\hline Disabled & $1(1.1)$ & $0(0.0)$ & $1(2.0)$ & .38 \\
\hline Unemployed & $27(30.7)$ & $7(18.4)$ & $20(40.0)$ & $<.05$ \\
\hline Insurance type, No. (\%) & & $215(50.0)$ & $215(50.0)$ & \\
\hline BlueCross & $74(17.2)$ & $28(13.0)$ & $46(21.4)$ & $<.05$ \\
\hline BlueShield & $86(20.0)$ & $36(16.7)$ & $50(23.3)$ & $<.05$ \\
\hline Commercial & $58(13.5)$ & $20(9.3)$ & $38(17.7)$ & $<.01$ \\
\hline Government programs & $13(3.0)$ & $7(3.3)$ & $6(2.8)$ & .39 \\
\hline $\mathrm{HMO}$ & $15(3.5)$ & $7(3.3)$ & $8(3.7)$ & .40 \\
\hline Medicaid & $149(34.7)$ & $99(46.0)$ & $50(23.3)$ & $<.01$ \\
\hline Medicare & $4(0.9)$ & $3(1.4)$ & $1(0.5)$ & .16 \\
\hline Self pay & $20(4.7)$ & $12(5.6)$ & $8(3.7)$ & .18 \\
\hline $\mathrm{PPO}$ & $5(1.2)$ & $0(0.0)$ & $5(2.3)$ & $<.05$ \\
\hline Unknown & $6(1.4)$ & $3(1.4)$ & $3(1.4)$ & .50 \\
\hline
\end{tabular}

GED, General Educational Development; HMO, Health Maintenance Organization; PPO, Preferred Provider Organization; FM, Family Medicine; OB/Gyn, Obstetrics and Gynecology.

cohort was lower compared with OB/Gyn (61\% vs $71 \% ; P=.03)$, although there was a larger proportion of unknown or undocumented occupations among the FM cohort $(21 \%$ vs $6 \% ; P<.00001)$. There was a larger proportion of FM patients that were insured through Medicaid when compared with patients seen by OB/Gyn ( $46 \%$ vs $23 \%$; $P<.00001)$. Finally, the average age for the FM cohort was 27 years old (SD, 5.17) compared with 29 years old $(\mathrm{SD}, 5.60)$ for the OB/Gyn group $(P<.0001)$. Figure 2 visually depicts the significant differences between the cohorts in terms of education, race, health insurance, and marital status.

\section{Social and Medical History}

The FM cohort was more likely to report prepregnancy recreational drug use $(11 \%$ vs $5 \% ; P<.05)$, prepregnancy tobacco use $(27 \%$ vs $18 \% ; P=.02)$, and tobacco use during pregnancy compared with $\mathrm{OB} / \mathrm{Gyn}(17 \%$ vs $8 \% ; P<.01)$. There were no statistically significant differences between the cohorts in reporting of intimate partner violence. 
Figure 2. Social determinants of health between FM and OB/Gyn for PNC patients. Abbreviations: FM, Family Medicine; OB/Gyn, Obstetrics and Gynecology; PNC, Prenatal Care.
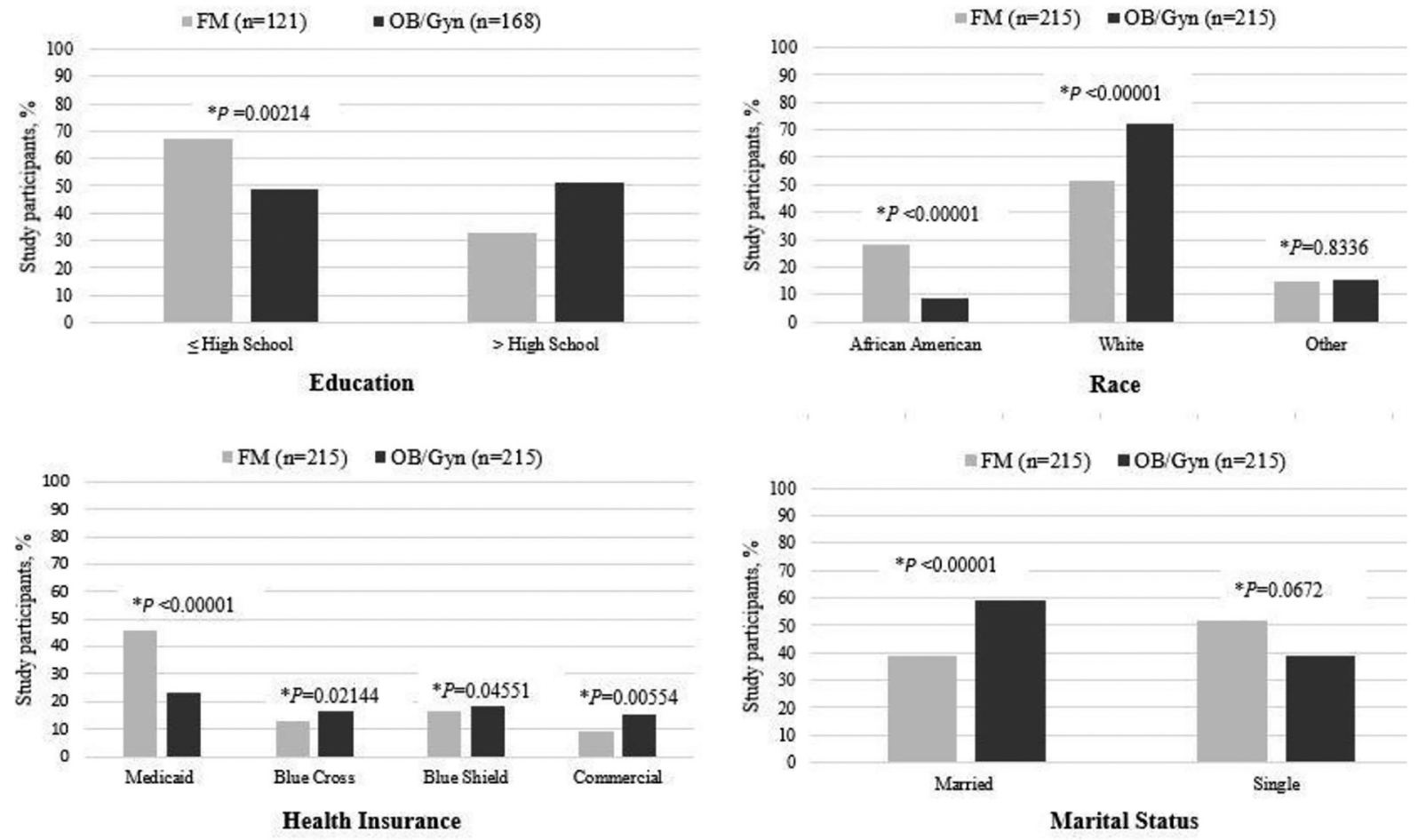

PNC

There was no significant difference between the 2 cohorts related to gravidity (Table 2). In addition, no significant differences were noted between the groups for birth control use at conception $(6 \%$ vs $5 \% ; P=.66)$, adherence to immunization schedule during pregnancy $(79 \%$ vs $81 \% ; P=.63)$, nonadherence to prenatal visit schedule ( $9 \%$ vs $7 \% ; P=.60$ ), average Body Mass Index at first visit (28 vs $27 \mathrm{~kg} / \mathrm{m}^{2}$; $P=.12$ ), or prenatal sexually transmitted infection rates $(5 \%$ vs $3 \% ; P=.24)$. The mean interconception period for $\mathrm{FM}(\mathrm{n}=32)$ was 16 months and 14 months for OB/Gyn $(n=40)$ showing no statistically significant difference $(P=.39)$.

\section{Pregnancy Outcomes}

FM patients had a lower rate of total Cesarean sections (C-section), including primary and repeat, when compared with OB/Gyn (23\% vs $32 \%$; $P=.04)$. Of note, the rate of primary $\mathrm{C}$-section was not statistically different for FM when compared with OB/Gyn ( $13 \%$ vs $19 \%$; $P=.09)$. The predominant indications for primary $\mathrm{C}$-section among both cohorts included arrest of dilation $(n=15)$, arrest of descent $(n=5)$, and nonreassuring fetal heart rate

tracing $(\mathrm{n}=11)$ without any significant differences between the cohorts. When compared with $\mathrm{OB} /$ Gyn, infants born to the FM cohort on average weighed $135 \mathrm{~g}$ less $(P=.01)$. There was no statistically significant difference in the average gestational age in weeks at delivery for FM when compared with $\mathrm{OB} / \mathrm{Gyn}(38.4$ vs $38.7 ; P=.21)$.

\section{Discussion}

This retrospective chart review found that prenatal patients cared for by FM suffered a greater burden to their health from a $\mathrm{SDoH}$ perspective compared with $\mathrm{OB} / \mathrm{Gyn}$ at our institution. FM prenatal patients were more likely to be African American, younger, have less than or equal to a high school education, be single, use Medicaid, have used recreational drugs or tobacco before pregnancy, and continue tobacco use during pregnancy. Conversely, OB/Gyn prenatal patients were more likely to be White, have higher levels of education, be married, and consume alcohol before pregnancy. Despite the numerous social challenges facing prenatal patients cared for by FM, there was no statistically significant difference in gestational age at delivery and the rate of total $\mathrm{C}$-sections was lower when compared with $\mathrm{OB} / \mathrm{Gyn}$. When 
compared with the OB/Gyn cohort, infants in the FM cohort had lower birth weights. There was also no statistically significant difference in adherence to immunization schedule during pregnancy or adherence to prenatal visit schedule between cohorts. When considering the higher social vulnerability of the prenatal patients cared for by FM at our institution, we would argue that the care provided by FM as compared with $\mathrm{OB} / \mathrm{Gyn}$ was of higher impact.

Despite the richness of data surrounding the disparity between race and pregnancy outcomes, maternal mortality for African-American women remains 1 of our nation's greatest public health inequalities. ${ }^{15,23}$ The FM cohort comprised a statistically significant greater proportion of AfricanAmerican women and yet the only identified disparity in outcomes was a slightly decreased average birth weight when compared with the OB/Gyn cohort. International data support the correlation between higher rates of $\mathrm{C}$-section deliveries and maternal mortality rates. ${ }^{24}$ Our results demonstrate overall lower C-section rates within the FM cohort when compared with the OB/Gyn cohort, further highlighting evidence of risk reduction despite the added complexities of the patient population.

Furthermore, the larger number of AfricanAmerican patients cared for by FM, as compared with $\mathrm{OB} / \mathrm{Gyn}$, may be secondary to geography as multiple FM offices at our institution are located in regions where there are proportionally more African American residents. These findings are consistent with previous physician distribution studies by specialty showing FM physicians are the largest source of providers in rural areas ${ }^{25}$ and a large number of providers in urban areas, further emphasizing the need for FM physicians to provide PNC. ${ }^{26}$

Our data suggest that FM physicians care for more vulnerable prenatal patients and yet the number of FM physicians who provide this care nationally, is decreasing. While the inclusion of PNC in practice has been found to significantly decrease the rate of burnout for FM physicians, ${ }^{27}$ only $13 \%$ of graduates actually include this in their practice. ${ }^{28}$ Previously documented barriers to FM physicians performing PNC and deliveries included lack of interest, lack of opportunities in practice, and lifestyle considerations. ${ }^{2,5}$ It has been proposed that the avenue for targeting this deficiency is to reform FM education to include more maternity care experience, likely through extending length of training, and targeting rural areas of practice that have less access to Obstetricians. ${ }^{29}$

A limitation of this study was the number of unknown or undocumented demographic information within the FM cohort (Table 1). We suspect a contributor to this is that FM physicians at our institution fill out the entirety of the ACOG form themselves as opposed to nursing staff with $\mathrm{OB} /$ Gyn. Addition of these missing documentation elements in the FM cohort may alter various outcomes resulting in potential differences in the groups where currently no difference exists, such as birth control use at conception. We also noted providerto-provider variation in recording certain ACOG elements of social and medical history. For example, some providers mark positive any past trauma/ violence without specifying the type, including intimate partner violence. This may have complicated

Table 2. PNC and Delivery Outcomes of FM and OB/Gyn Cohorts

\begin{tabular}{lccc}
\hline & Family Medicine & Obstetrics and Gynecology & $P$ Value \\
\hline Overall, No. (\%) & $215(50.0)$ & $215(50.0)$ & - \\
Gravidity, mean (SD) & $2.57(1.7)$ & $2.43(1.7)$ & .18 \\
Mode of delivery, No (\%) & & & \\
pCS & $28(13.0)$ & $41(19.1)$ & .09 \\
rCS & $21(9.8)$ & $27(12.6)$ & .36 \\
Cesarean section total & $49(22.8)$ & $68(31.6)$ & .05 \\
SVD & $120(55.8)$ & $122(56.7)$ & .85 \\
Birth weight, g, mean (SD) & $3220(618)$ & $3356(545)$ & .21 \\
Gestational age at delivery, weeks, mean (SD) & $38.4(2.7)$ & $18(8.4)$ & $<.01$ \\
Tobacco use during pregnancy, No. (\%) & $36(16.7)$ & \\
\hline
\end{tabular}

pCS, primary cesarean section; rCS, Repeat cesarean section; SVD, spontaneous vaginal delivery; FM, Family Medicine; OB/Gyn, Obstetrics and Gynecology; PNC, Prenatal Care; SD, standard deviation. 
and made inaccurate at least this 1 comparison point, as we saw a significantly greater number of unknowns marked in the FM cohort compared with OB/Gyn for intimate partner violence $(13.9 \%$ vs $2.32 \%, P<.00001)$.

In all, our work demonstrates that when compared with OB/Gyn at our institution, FM physicians provide PNC to a cohort of patients with an increased burden of social risk without compromise to care as evidenced by a lower $\mathrm{C}$-section rate and similar gestational age at delivery.

\section{Conclusions}

At our institution, we found that FM provided PNC to a cohort of patients with a greater burden of SDoH as compared with OB/Gyn without compromising quality of PNC or pregnancy-related outcomes. We hope our findings help guide resource allocation to FM physicians caring for highly vulnerable patient populations. Patient-centered resource allocations might include the construction of maternity centers in rural areas where FM physicians practice, and development of interdisciplinary PNC medical homes that include social work and mental health resources. Similarly, hiring additional staff to administer PNC questionnaires that assess barriers to care through the lens of SDoH could help provide patients with necessary social resources at the onset of pregnancy. Provider-centered resource allocations previously proposed have included the provision of malpractice insurance by employers and call sharing. ${ }^{30}$ We further suggest reviewing reimbursement models to incentivize the practice of obstetrics by FM physicians, streamlining the obstetric credentialing process, and fostering structured mentorship for newly graduated FM physicians to prevent the devel-

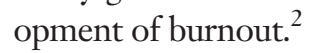

In conclusion, our study should serve to encourage changes to residency training to promote PNC and obstetrics within FM to fill the growing obstetric provider shortage. We recognize that this will require a health system wide culture shift wherein the barriers that plague the ability of FM physicians to provide PNC and obstetric care are eliminated and opportunities for FM physicians to provide this care are garnished. As the body of evidence grows demonstrating the impact of SDoH on a person's health, studies like this 1 provide evidence to the value of investing time and resources into FM physicians providing PNC and obstetric care.
We acknowledge Larry Leo for his assistance with data extraction. We thank Robert Lennon, MD; and our department chair, Mack Ruffin IV, MD, MPH; for their consultation and review of the manuscript.

To see this article online, please go to: http://jabfm.org/content/ 34/1/181..full.

\section{References}

1. Macinko J, Starfield B, Shi L. Quantifying the health benefits of primary care physician supply in the United States. Int J Health Serv 2007;37: 111-26.

2. Barreto TW, Eden A, Hansen ER, Peterson LE. Opportunities and barriers for family physician contribution to the maternity care workforce. Fam Med 2019;51:383-8.

3. Barreto T, Peterson LE, Petterson S, Bazemore AW. Family physicians practicing high-volume obstetric care have recently dropped by one-half. Am Fam Physician 2017;95:762.

4. Rayburn WF. The obstetrician/gynecologist workforce in the United States: facts, figures, and implications 2017. Washington, DC: American Congress of Obstetricians and Gynecologists; 2017.

5. American Academy of Family Physicians. Family medicine facts. Table 11: clinical services performed by physicians. 2018. Available from: https:// www.aafp.org/about/the-aafp/family-medicinespecialty/facts/table-11(rev).html. Accessed July 27, 2020.

6. Cohen D, Coco A. Declining trends in the provision of prenatal care visits by family physicians. Ann Fam Med 2009;7:128-33.

7. Xierali IM, Nivet MA, Rayburn WF. Relocation of obstetrician-gynecologists in the United States, 2005-2015. Obstet Gynecol 2017;129:543-50.

8. Rayburn $W$. Who will deliver the babies? Identifying and addressing barriers. J Am Board Fam Med 2017; 30:402-4.

9. Braveman P, Egerter S, Williams DR. The social determinants of health: coming of age. Annu Rev Public Health 2011;32:381-98.

10. Penman-Aguilar A, Talih M, Huang D, Moonesinghe R, Bouye K, Beckles G. Measurement of health disparities, health inequities, and social determinants of health to support the advancement of health equity. J Public Health Manag Pract 2016;22:S33-S42.

11. Adler N, Stewart J. The biology of disadvantage: socioeconomic status and health. Ann N Y Acad Sci 2010;1186:1-275.

12. Secretary's Advisory Committee on Health Promotion and Disease Prevention Objectives for 2020. Healthy People 2020: An Opportunity to Address the Societal Determinants of Health in the United States. 2010. 
Available from: https://www.healthypeople.gov/sites/ default/files/SocietalDeterminantsHealth.pdf.

13. Gadson A, Akpovi E, Mehta PK. Exploring the social determinants of racial/ethnic disparities in prenatal care utilization and maternal outcome. Semin Perinatol 2017;41:308-17.

14. Leonard SA, Main EK, Scott KA, Profit J, Carmichael SL. Racial and ethnic disparities in severe maternal morbidity prevalence and trends. Ann Epidemiol 2019;33:30-6.

15. Nelson DB, Moniz MH, Davis MM. Populationlevel factors associated with maternal mortality in the United States, 1997-2012. BMC Public Health 2018;18:1007.

16. De Graaf JP, Steegers EA, Bonsel GJ. Inequalities in perinatal and maternal health. Curr Opin Obstet Gynecol 2013;25:98-108.

17. Mydam J, David R, Rankin KM, Collins JW. Low birth weight among infants born to Black Latina women in the United States. Matern Child Health J 2019;23:538-46.

18. Ju AC, Heyman MB, Garber AK, Wojcicki JM. Maternal obesity and risk of preterm birth and low birthweight in Hawaii PRAMS, 2000-2011. Matern Child Health J 2018;22:893-902.

19. Graham R, Roberts RG, Ostergaard DJ, Kahn NB, Pugno PA, Green LA. Family practice in the United States: a status report. JAMA 2002;288:1097-101.

20. Clements DS. Social determinants of health in family medicine residency education. Ann Fam Med 2018;16:178.

21. American Academy of Pediatrics and The American College of Obstetrics and Gynecologists. Guidelines for perinatal care. 8th ed. Washington, DC: American
Academy of Pediatrics and the American College of Obstetricians and Gynecologists; 2017.

22. Srinivasan S, Schlar L, Rosener SE, et al. Delivering interconception care during well-child visits: an IMPLICIT Network study. J Am Board Fam Med 2018;31:201-210.

23. Louis JM, Menard MK, Gee RE. Racial and ethnic disparities in maternal morbidity and mortality. Obstet Gynecol 2015;125:690-694.

24. Mascarello KC, Horta BL, Silveira MF. Maternal complications and cesarean section without indication: systematic review and meta-analysis. Rev Saude Publica 2017;51:105.

25. AHRQ. The Distribution of the U.S. Primary Care Workforce. Primary Care Workforce Facts and Stats No. 3. Available from: https://www.ahrq.gov/ research/findings/factsheets/primary/pcwork3/index. html. Accessed April 27, 2020.

26. Rosenblatt RA, Hart LG. Physicians and rural America. West J Med 2000;173:348-351.

27. Weidner AKH, Phillips RL, Jr, Fang B, Peterson LE. Burnout and scope of practice in new family physicians. Ann Fam Med 2018;16:200-205.

28. Barreto TW, Eden AR, Hansen ER, Peterson LE. Barriers faced by family medicine graduates interested in performing obstetric deliveries. J Am Board Fam Med 2018;31:332-333.

29. Young RA, Sundermeyer L. Family medicine and obstetrics: let's stop pretending. J Am Board Fam Med 2018;31:328-331.

30. Eden AR, Barreto T, Hanse ER. Experiences of new family physicians finding jobs with obstetrical care in the USA. Fam Med Community Health 2019; 7:e000063. 